\title{
Altitude dependence of fluorescence light emission by extensive air showers
}

\author{
B. Keilhauer ${ }^{\mathrm{a}, *}$ J. Blümer ${ }^{\mathrm{a}, \mathrm{b}}$ R. Engel ${ }^{\mathrm{b}}$ H.O. Klages ${ }^{\mathrm{b}}$ \\ ${ }^{a}$ Universität Karlsruhe, Institut für Experimentelle Kernphysik, 76021 Karlsruhe, Germany \\ ${ }^{\mathrm{b}}$ Forschungszentrum Karlsruhe, Institut für Kernphysik, 76021 Karlsruhe, Germany
}

\begin{abstract}
Fluorescence light is induced by extensive air showers while developing in the Earth's atmosphere. The number of emitted fluorescence photons depends on the conditions of the air and on the energy deposited by the shower particles at every stage of the development. In a previous model calculation, the pressure and temperature dependences of the fluorescence yield have been studied on the basis of kinetic gas theory, assuming temperature-independent molecular collision cross-sections. In this work we investigate the importance of temperature-dependent collision cross-sections and of water vapour quenching on the expected fluorescence yield. The calculations will be applied to simulated air showers while using actual atmospheric profiles to estimate the influence on the reconstructed energy of extensive air showers.
\end{abstract}

Key words: fluorescence yield, altitude dependence, atmosphere, extensive air showers, ultra-high energy cosmic rays PACS: 96.50.sd, 96.50.sb, 33.50.-j

\section{Introduction}

Several air shower experiments like HiRes [1, the Pierre Auger Observatory [2, and Telescope Array [3], are using the fluorescence technique for detecting extensive air showers (EAS) induced by ultra-high energy cosmic rays. Measuring the fluorescence light that nitrogen molecules emit after being excited by charged particles of EAS is currently the most direct method for determining the energy of EAS in a model-independent way. A thorough understanding of the light emission process is necessary to obtain the primary energy of EAS with high precision.

In this paper, we extend our previous model calculation for the fluorescence light emission [4] by including the latest results on input parameters and their temperature dependence as obtained in labo-

\footnotetext{
* bianca.keilhauer@ik.fzk.de
}

ratory measurements. For the reconstruction of air shower events, the light emission has to be known in dependence on altitude in the Earth's atmosphere at which the shower is observed. Up to now, the altitude dependence has been considered by including air density profiles and collisional quenching of nitrogen-nitrogen and nitrogen-oxygen molecules as described by kinetic gas theory. The cross-sections for collisional quenching were taken to be temperature independent. However, the cross-sections describing collisional quenching are known to be temperature-dependent [5. Grün and Schopper [5] found a decreasing collisional quenching crosssection with increasing temperature. Recently, the AirFly experiment has studied collisional quenching cross-sections in dependence on temperature [6]. These data are also included in the model calculations presented in this article. In addition we investigate the influence of water vapour on the fluorescence yield, using relative humidity measure- 
ments performed at the site of the Auger detector in Argentina.

\section{Model calculation and experimental data}

Following the mathematical description in 4], the fluorescence yield (number of photons of wavelength $\lambda$ produced per meter track length) is written as

$F Y_{\lambda}=\frac{\varepsilon_{\lambda}^{0}}{1+\left(p / p_{\nu^{\prime}}^{\prime}(T)\right)} \cdot \frac{\lambda}{h c} \cdot \frac{d E}{d X} \cdot \rho_{a i r}$,

with $\varepsilon_{\lambda}^{0}$ being the fluorescence efficiency at wavelength $\lambda$ without collisional quenching, $p$ is air pressure, $p_{\nu^{\prime}}^{\prime}$ is a reference pressure at which the mean life time of the radiative transition to any lower state $\tau_{0}$ is equal to that of collisional quenching $\tau_{c}$. The index $\nu^{\prime}$ indicates the excitation level of a band system. The air density is given by $\rho_{\text {air }}$ and the energy deposited locally by a charged particle of an EAS is $\frac{d E}{d X}$.

Assuming air to be a two-component gas, the relation between $p$ and $p_{\nu^{\prime}}^{\prime}$ is given by

$$
\begin{aligned}
& \frac{p}{p_{\nu^{\prime}}^{\prime}}=\frac{\tau_{0, \nu^{\prime}} p_{\mathrm{air}} \cdot N_{A}}{R \cdot T} \cdot \sqrt{\frac{k T N_{A}}{\pi}} \\
& \quad \cdot\left(4 \cdot C_{v}\left(\mathrm{~N}_{2}\right) \cdot \sigma_{\mathrm{NN}, \nu^{\prime}}(T) \cdot \sqrt{\frac{1}{M_{m, \mathrm{~N}}}}\right. \\
& \left.\quad+2 \cdot C_{v}\left(\mathrm{O}_{2}\right) \cdot \sigma_{\mathrm{NO}, \nu^{\prime}}(T) \cdot \sqrt{2\left(\frac{1}{M_{m, \mathrm{~N}}}+\frac{1}{M_{m, \mathrm{O}}}\right)}\right),
\end{aligned}
$$

with the masses per mole for nitrogen $M_{m, \mathrm{~N}}$ and oxygen $M_{m}$, O and the fractional part per volume $C_{v}$ of the two gas components. The temperature dependence of the collisional quenching cross-sections is parametrised as

$\sigma_{\mathrm{Nx}, \nu^{\prime}}(T)=\sigma_{\mathrm{Nx}, \nu^{\prime}}^{0} \cdot T^{\alpha_{\nu^{\prime}}}$.

For example, in case of the AirFly experiment, it is given by $\sigma_{\mathrm{Nx}, \nu^{\prime}}^{0}=\sigma_{\mathrm{Nx}, \nu^{\prime}} \cdot 293^{-\alpha_{\nu^{\prime}}}$. The crosssections have been measured for the bands at $313.6 \mathrm{~nm}(2 \mathrm{P}(2-1)), 337.1 \mathrm{~nm}(2 \mathrm{P}(0-0)), 353.7 \mathrm{~nm}$ $(2 \mathrm{P}(1-2))$, and $391.4 \mathrm{~nm}(1 \mathrm{~N}(0-0))$. In the calculations presented here, it is assumed that $\alpha_{\nu^{\prime}}$ is the same for all bands within its band system. In the AirFly experiment, $\alpha_{\nu^{\prime}}$ have been measured for dry air and no differentiation has been made for nitrogen or oxygen. For fitting those data into the model calculation, it is assumed that these $\alpha$-coefficients can be applied to the quenching cross-sections of both N-N and N-O collisions.
An absolute calibration of the AirFly experiment has not yet been published. Therefore, the fluorescence efficiency $\epsilon_{337.1 \mathrm{~nm}}^{0}$ has been set to $0.082 \%$ of the deposited energy, the value given by Bunner [7. The same normalisation for $\epsilon_{337.1 \mathrm{~nm}}^{0}$ is used in the calculations of [4].

In the following we will show two model calculations based on AirFly measurements. If the temperature dependence of the collisional quenching crosssections is not considered, Eq. (1) is used with the parameters $p_{\nu^{\prime}}^{\prime}$ given in [8]. Eq. (2) is applied for calculating the fluorescence yield with temperaturedependent cross-sections.

The resulting fluorescence yield spectrum, calculated for the US Standard Atmosphere at sea level, can be seen in Fig. 1 in comparison with calculations and other measurements presented in detail in [4]. The calculations following the mathemati-

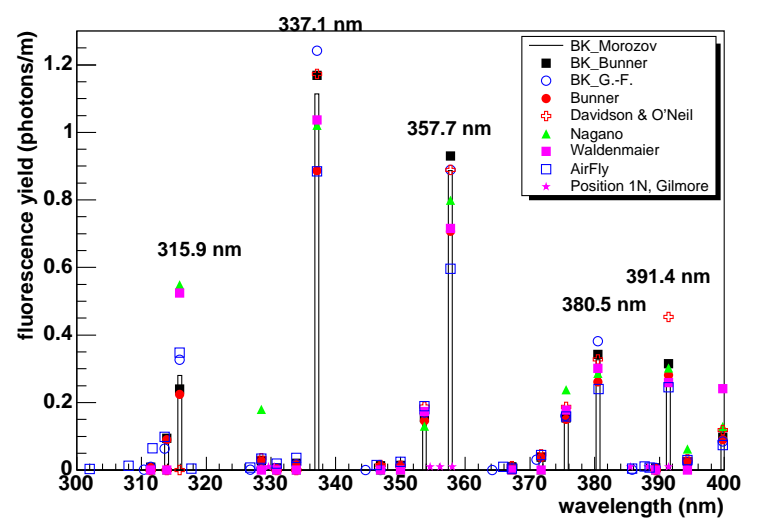

Fig. 1. Fluorescence yield spectra of several calculations and measurements for $0.85 \mathrm{MeV}$ electrons as exciting particles in the US Standard Atmosphere at sea level. The calculations following the mathematical description in 4] are labelled with BK_name, where BK stands for the initials of the corresponding author of [4] and name indicates the authors of the input parameters used. Details can be found in the text. The bars indicate the combination of calculation and input parameters which is favoured in [4].

cal description in 4] are labelled with BK_name, where BK stands for the initials of the corresponding author of [4] and name indicates the authors of the input parameters used. Input parameters in Eq. 2 are the deactivation constants which are the radiative life time $\tau_{0, \nu^{\prime}}$ and the collisional crosssection between nitrogen and nitrogen molecules $\sigma_{\mathrm{NN}, \nu^{\prime}}$ and between nitrogen and oxygen molecules $\sigma_{\mathrm{NO}, \nu^{\prime}}$. Bunner provides collisional cross-sections and radiative life times for the most prominent band systems of nitrogen [7]. Using these input pa- 
rameters in the calculation, the results are labelled with BK_Bunner. Recent measurements by Morozov et al. 9] were performed for the $2 \mathrm{P} \nu^{\prime}=0,1$ band systems. For results named BK_Morozov, the values from Bunner are replaced by the newer data by Morozov et al. where available. An alternative calculation of the fluorescence efficiency without collisional quenching $\epsilon_{\lambda}^{0}$ is also presented in 4]. Here the Einstein coefficients $A_{\nu^{\prime} \nu^{\prime \prime}}$ and the radiative life times from Gilmore et al. [10] and the relative apparent excitation cross-section $Q_{\text {app }}$ from Fons et al. 11] are used. For details of this procedure see 4 . The resulting fluorescence yield is labelled with BK_G.-F.

For a relative comparison of 19 bands between 300 and $400 \mathrm{~nm}$ see Fig. 2. The basis is the calculation

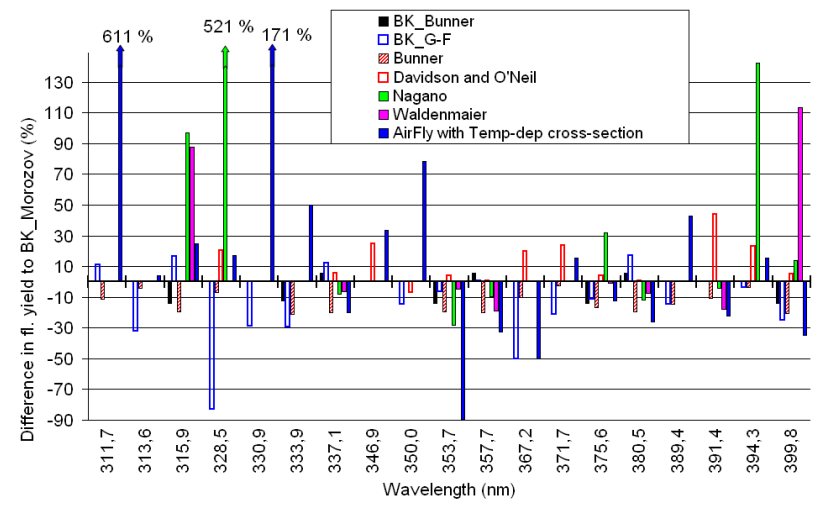

Fig. 2. Relative comparison of 19 bands of the "BK_Morozov"-calculation with measurements and further calculations. The absolute fluorescence yield of these contributions can be seen in Fig. 1 with the same colour labels.

"BK_Morozov".

\section{Temperature-dependent cross-section}

In the following, the fluorescence yield for $0.85 \mathrm{MeV}$ electrons and for EAS are studied using the "BK_Morozov"-calculation upgraded by temperature-dependent collisional quenching crosssections.

The fluorescence yield profiles for a $0.85 \mathrm{MeV}$ electron, corresponding to an energy deposit of $0.1677 \mathrm{GeV}$ per $\mathrm{g} / \mathrm{cm}^{2}$, is shown in Fig. 3 for the US Standard Atmosphere. The yield is the sum of 26 bands between 300 and $400 \mathrm{~nm}$ as listed in 8 . The solid black line represents the results where no temperature-dependent collisional quenching crosssections have been considered. The red dashed line includes the temperature-dependent cross-section

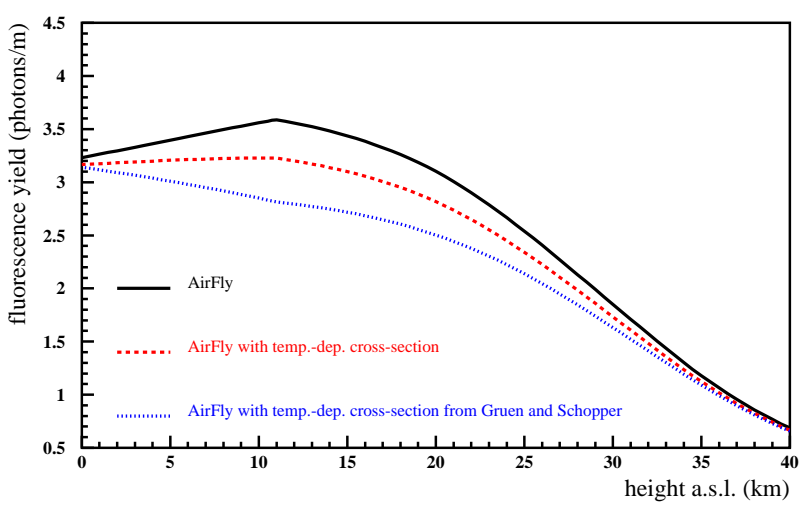

Fig. 3. Fluorescence yield profiles for a $0.85 \mathrm{MeV}$ electron in the US Standard Atmosphere with measured parameters from the AirFly experiment 86 . The yield is the sum of 26 bands between 300 and $400 \mathrm{~nm}$ as listed in 8 . See text for details.

as measured by AirFly. The blue dotted line reflects the AirFly data where the $\alpha$-coefficients for the temperature-dependent cross-sections are replaced by the data from Grün and Schopper [5]. The value has been extracted from Fig. 6 of $[5]$ and is therefore quite imprecise. Additionally, a caveat has to be applied to this comparison: Grün and Schopper measured one $\alpha$-coefficient for the entire wavelength range in pure nitrogen and not in air. For this simple comparison, the value for N-N collisions has been used for the $p^{\prime}$ of air. However, the AirFly result confirms a decreasing cross-section with increasing temperature and also the absolute scales of the two publications are in quite good agreement. The new

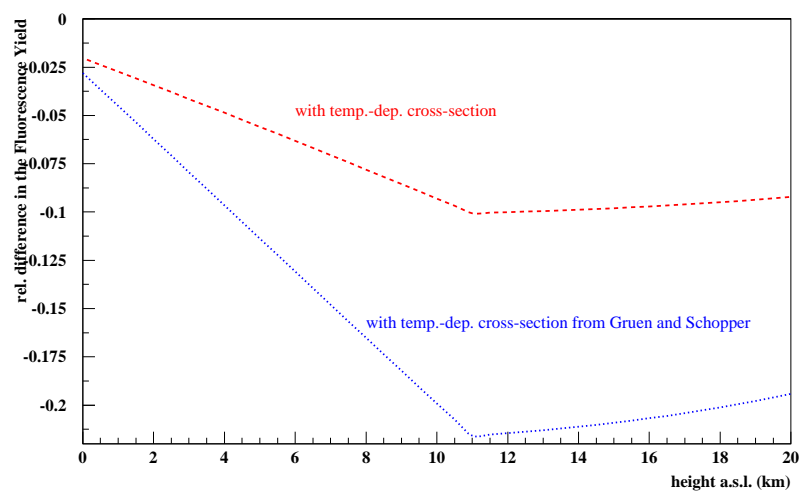

Fig. 4. Relative difference of the fluorescence yield calculated with temperature-dependent collisional cross-sections compared with the former model calculation.

dependence reduces the fluorescence yield at sea level by about $2 \%$ compared with the former model calculation, see Fig 4. This deficit increases with 
increasing altitude to about $10 \%$ at $11 \mathrm{~km}$ a.s.l. in case of AirFly and to about $20 \%$ in case of Grün and Schopper data.

In the next step, the calculation of the fluorescence yield is performed using Argentine atmospheres as given in [12]. The former model calculation leads to fluorescence yield profiles for the seasonal atmospheres as can be seen in Fig. 5] [4. Applying the temperature-dependent collisional

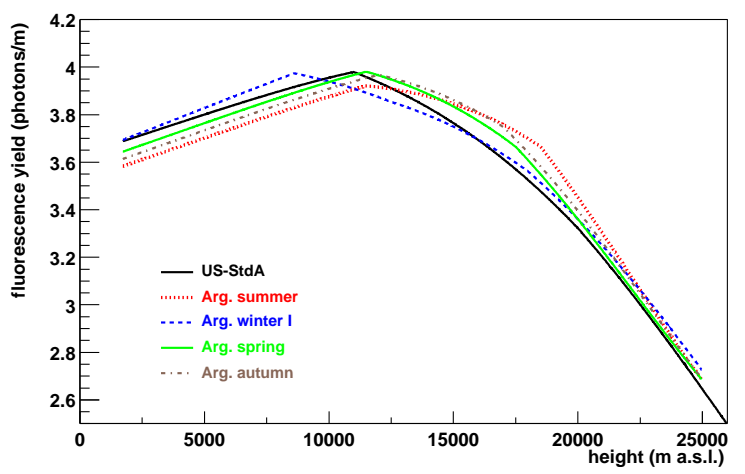

Fig. 5. Fluorescence yield profiles for a $0.85 \mathrm{MeV}$ electron in the US Standard Atmosphere and measured Argentine atmospheres as given in [12. The given yield is a sum of all emitted photons between 300 and $400 \mathrm{~nm}$.

cross-sections of AirFly in the calculations using Argentine atmospheres, the fluorescence yield profiles are strongly distorted, see Fig. 6. The different

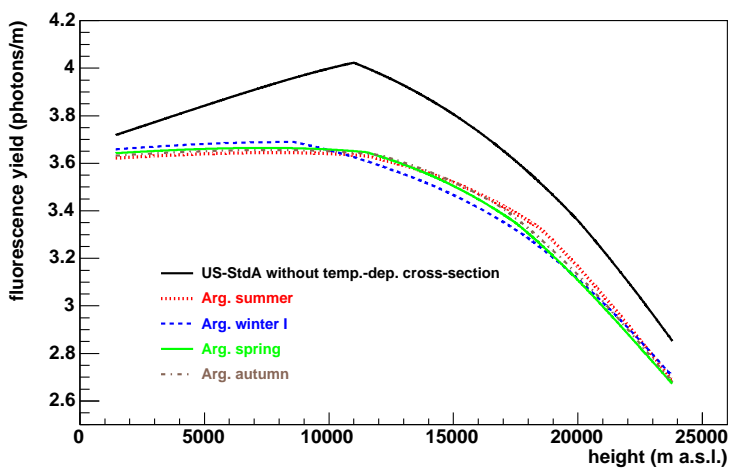

Fig. 6. Fluorescence yield profiles for a $0.85 \mathrm{MeV}$ electron in the US Standard Atmosphere and measured Argentine atmospheres as given in 12. The curves are calculated with the "BK_Morozov"-model of 4 combined with the temperature-dependent collisional quenching cross-sections from AirFly [6].

temperature profiles lead to a varying strength of the influences of the temperature-dependent cross-sections. In general, it can be stated that the temperature dependence reduces the increase of the fluorescence yield within the lowest $11 \mathrm{~km}$ in the atmosphere significantly. Furthermore, the differences between the seasonal atmospheres are reduced compared with the calculations without the temperature-dependent cross-sections. Fig. 7 displays the difference of the fluorescence yield in Argentine atmospheres to that in the US Standard Atmosphere with the former calculation.

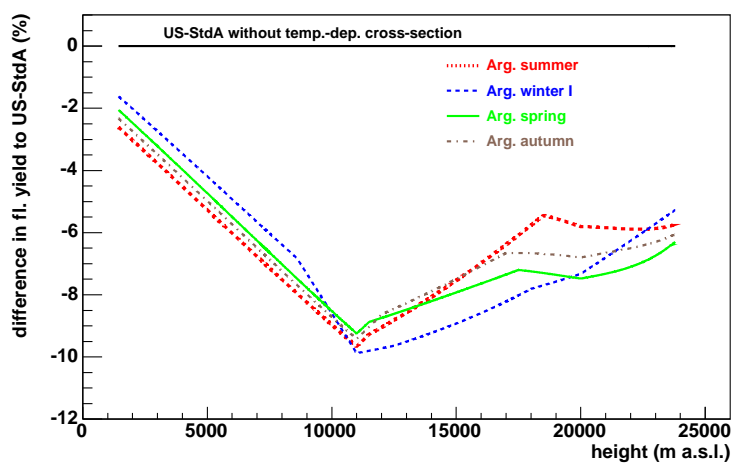

Fig. 7. Difference of the fluorescence yield profiles for a $0.85 \mathrm{MeV}$ electron in measured Argentine atmospheres to those in the US Standard Atmosphere without temperature-dependent collisional quenching cross-sections.

For estimating the importance of temperaturedependent cross-sections on reconstructing EAS profiles, two average iron-induced EAS with $E_{0}=$ $10^{19} \mathrm{eV}$ have been simulated with CORSIKA [13], one with vertical incidence and the other with $60^{\circ}$ inclination. The simulations have been performed with the US Standard Atmosphere and afterwards, the conversion from atmospheric depth $X$ to geometric altitude $h$ has been done applying Argentine atmospheres 12 . For these EAS profiles, the fluorescence light is calculated and shown in Fig. 8 for the vertical case and in Fig. 10 for the EAS with $60^{\circ}$ inclination. The corresponding differences of the fluorescence light in Argentine atmospheres to that in the US Standard Atmosphere are displayed in Figs. 9 and 11. The primary energy of EAS can be obtained be converting the fluorescence light into local energy deposit and then integrating over the entire profile of an EAS. Including temperaturedependent collisional quenching cross-section in the calculations, the expected shower light profile of an EAS with vertical incidence is reduced by $2.7 \%$ in Argentine summer, by $3.2 \%$ in autumn, by $3.7 \%$ in spring, and by $4.8 \%$ in winter. Doing the same for the $60^{\circ}$ inclined shower, the reduction of the 


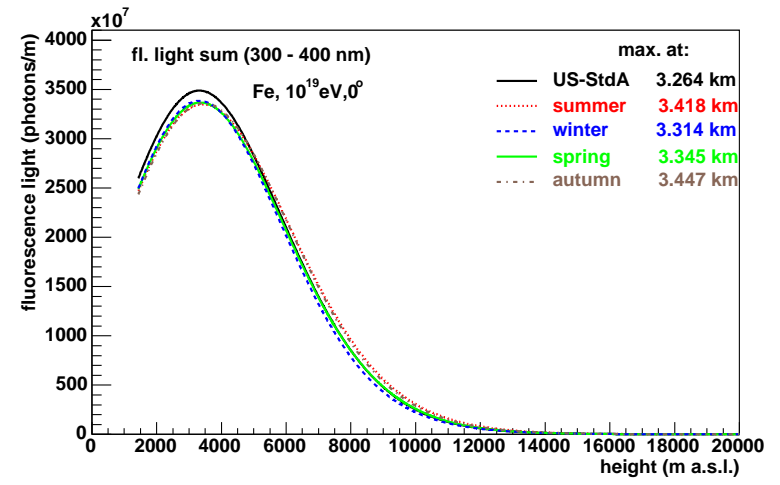

Fig. 8. Fluorescence light profiles for an iron-induced extensive air shower with $E_{0}=10^{19} \mathrm{eV}$ and vertical incidence in the US Standard Atmosphere and measured Argentine atmospheres as given in [12. The fluorescence emission is calculated with the "BK_Morozov"-model of 4] combined with the temperature-dependent collisional quenching cross-section from AirFly [6] and the extensive air shower is simulated with CORSIKA [13.

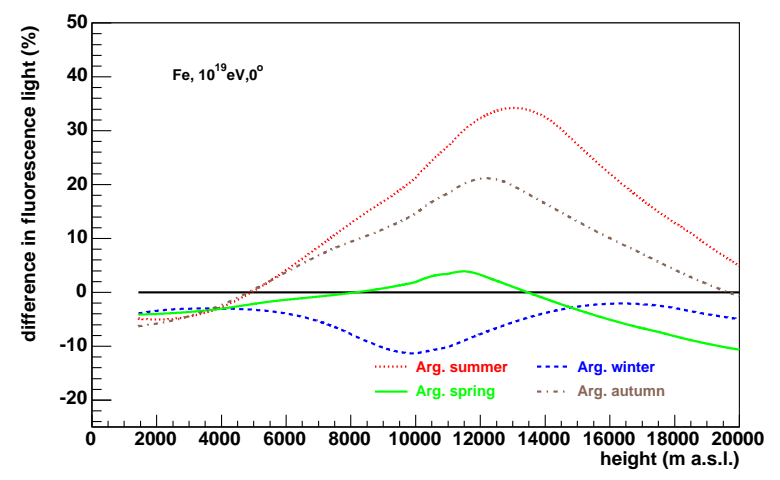

Fig. 9. Difference of the fluorescence light profiles as shown in Fig. 8

expected light increases to $6.3 \%$ in summer, $6.8 \%$ in autumn, $7.1 \%$ in spring, and $7.5 \%$ in winter. A former study has shown that varying atmospheric profiles influence the longitudinal shower development. It yields in slightly distorted profiles of the energy deposit of the air shower, which means an uncertainty of the energy reconstruction of the primary particle of less than 1\% [14. The position of the shower maximum has also been studied and a shift of about $-15 \mathrm{~g} \mathrm{~cm}^{-2}$ on average was found. Applying additionally the temperature-dependent cross-sections, the position of the shower maximum is only shifted slightly beyond it. In all atmospheric models, the additional shift is less than $50 \mathrm{~m}$.

The same calculations have been performed for proton-induced air showers with the same parame-

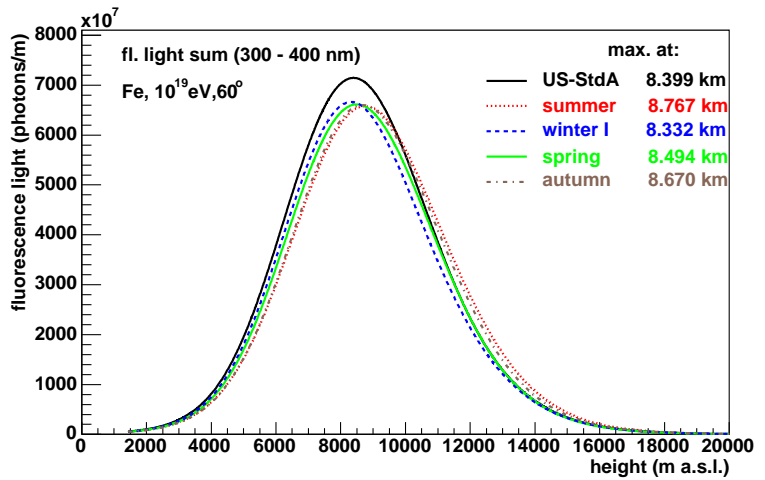

Fig. 10. Fluorescence light profiles for an iron-induced extensive air shower with $E_{0}=10^{19} \mathrm{eV}$ and $60^{\circ}$ inclination angle. Apart from the inclination, everything is the same as in Fig. 8

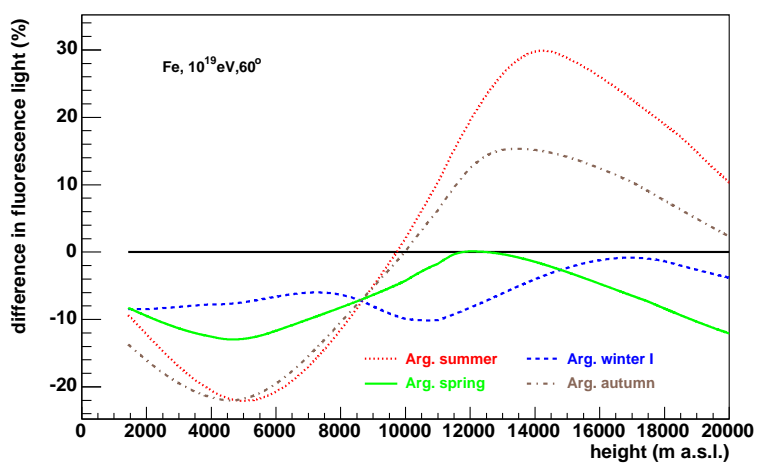

Fig. 11. Difference of the fluorescence light profiles as shown in Fig. 10

ters. The reduction of the expected light is increased by about $0.5 \%$ compared to the numbers of the ironinduced showers.

\section{Vapour quenching}

In this study, the model calculation has been expanded by including collisional quenching due to water vapour. An additional term is inserted in Eq. (2) to account for the collisions between nitrogen and water vapour molecules. The corresponding cross-section has been measured by e.g. Morozov et al. 9] and Waldenmaier 15. It has to be stressed that no temperature-dependence has been measured for the collisional quenching cross-section between water vapour and nitrogen. Typically in all atmospheric models, the humidity is set to zero. For this study, seasonal average profiles of relative humidity at the site of the Pierre Auger Observa- 
tory in Argentina, measured during night times, are fitted, see Fig. 12 .

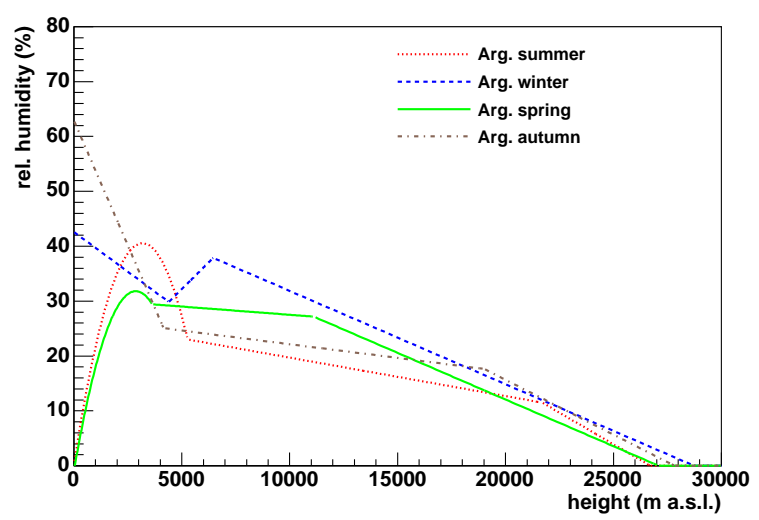

Fig. 12. Seasonal average profiles of relative humidity at the site of the Pierre Auger Observatory in Argentina as measured during night times.

Firstly, vapour quenching has been considered in the former model calculations applying the crosssections measured by Morozov et al. 9 without temperature-dependent cross-sections. The resulting fluorescence yield profiles for a $0.85 \mathrm{MeV}$ electron can be seen in Fig. 13. Vapour quenching re-

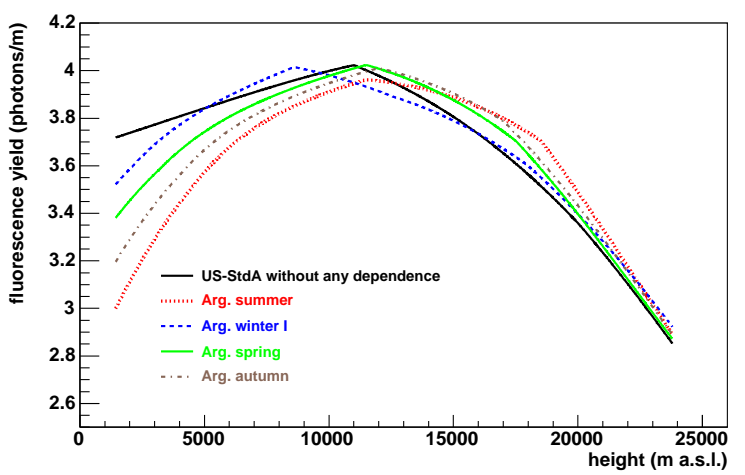

Fig. 13. Fluorescence yield profiles for a $0.85 \mathrm{MeV}$ electron in the US Standard Atmosphere and Argentine atmospheres. The fluorescence emission is calculated with the "BK_Morozov"-model of 4 including vapour quenching using the humidity profiles given in Fig. 12, however no temperature-dependent collisional quenching cross-section has been applied.

duces the fluorescence yield mainly in the lower part of the atmosphere, compare with Fig. 5 , because the water vapour content in the atmosphere is low at higher altitudes. The differences of the calculations in Argentine atmospheres to the US Standard Atmosphere are shown in Fig. 14. The effect is largest dur-

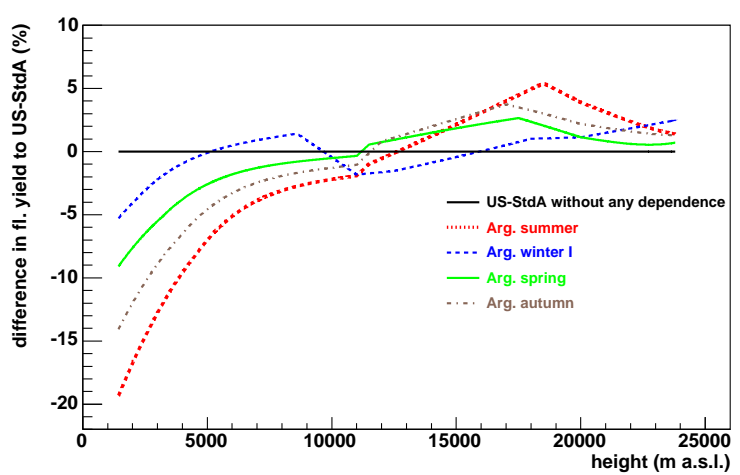

Fig. 14. Difference of the fluorescence yield profiles in Argentine atmospheres as shown in Fig. 13 to the US Standard Atmosphere.

ing summer, because more water vapour can be contained in warmer air. The reduction of fluorescence yield is most significant near the ground, about $20 \%$, and becomes less than $5 \%$ above $7 \mathrm{~km}$ a.s.l. During winter, the effect is of minor importance and only visible below about $3 \mathrm{~km}$ a.s.l.

Secondly, vapour quenching has been included in the model calculation presented in Sec. 3. Again, the fluorescence yield is determined for a $0.85 \mathrm{MeV}$ electron in Argentine atmospheres, Fig. 15. The dif-

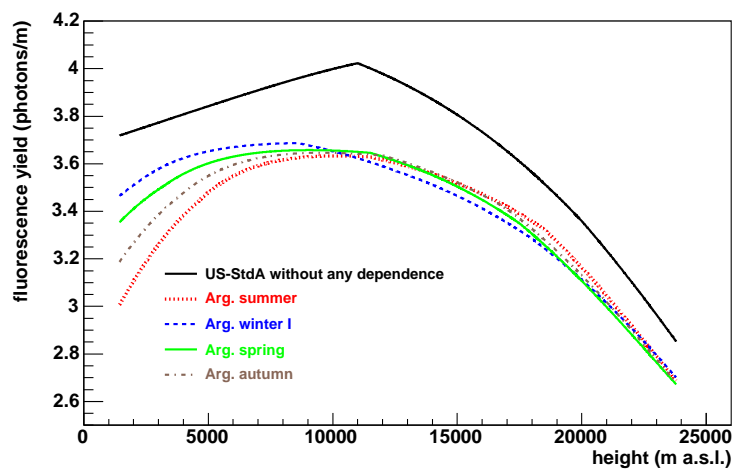

Fig. 15. Fluorescence yield profiles for a $0.85 \mathrm{MeV}$ electron in the US Standard Atmosphere and Argentine atmospheres. The fluorescence emission is calculated with the "BK_Morozov"-model of [4 including vapour quenching using the humidity profiles given in Fig. 12 and temperature-dependent cross-sections.

ference of the fluorescence yield in Argentine atmospheres to that in the US Standard Atmosphere can be seen in Fig. 16. The additional vapour quenching changes the profiles shown in Fig. 6 mainly in the lowest part of the atmosphere. In summer, the fluo- 


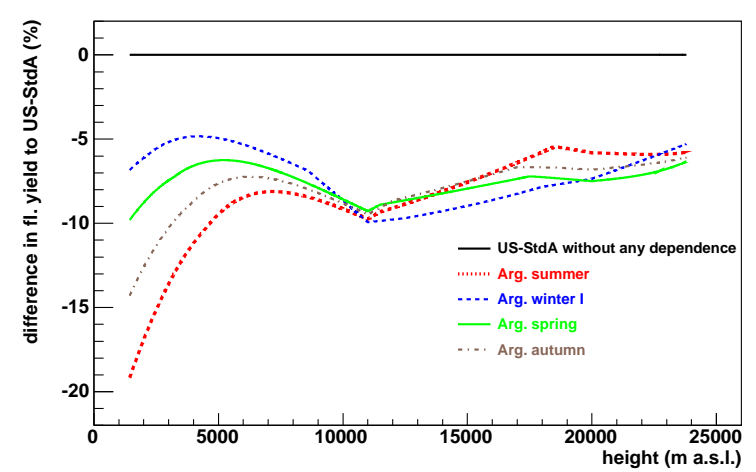

Fig. 16. Difference of the fluorescence yield profiles as shown in Fig. 15

rescence yield is reduced significantly and in winter the effect is smallest.

Thirdly, the model calculation including all dependences is applied to the average iron-induced EAS which are used already in Sec. 3. Since the effect of water vapour quenching is most important near ground, here only the fluorescence light profiles are shown for the vertical shower, Fig. 17 The

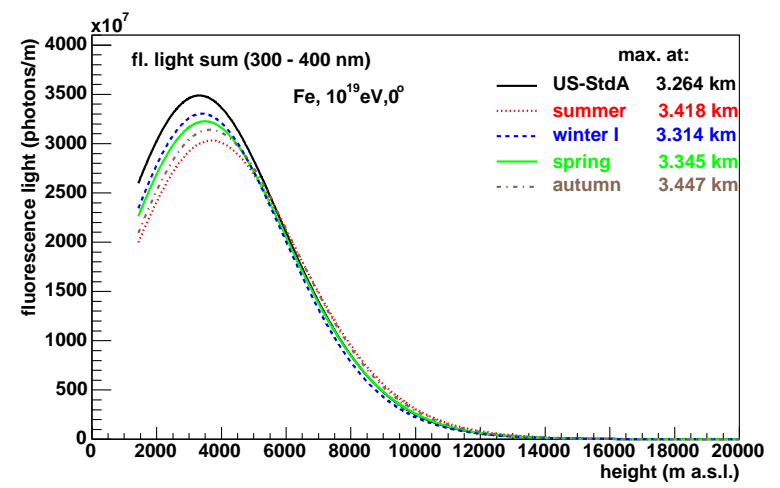

Fig. 17. Fluorescence light profiles for the iron-induced extensive air shower as shown in Fig. 8 For the fluorescence emission calculations, additionally the vapour quenching has been included.

graph of the differences of the fluorescence light in Argentine atmospheres to that in the US Standard Atmosphere can be seen in Fig. 18. The expected light of EAS is reduced by about additional $8.2 \%$ due to added water vapour quenching in Argentine summer, by $5.5 \%$ during autumn, by $3.4 \%$ during spring, and by about $2 \%$ during Argentine winter. In total, including the temperature-dependent collisional cross-sections and the water vapour quenching, the expected light of the EAS is reduced by

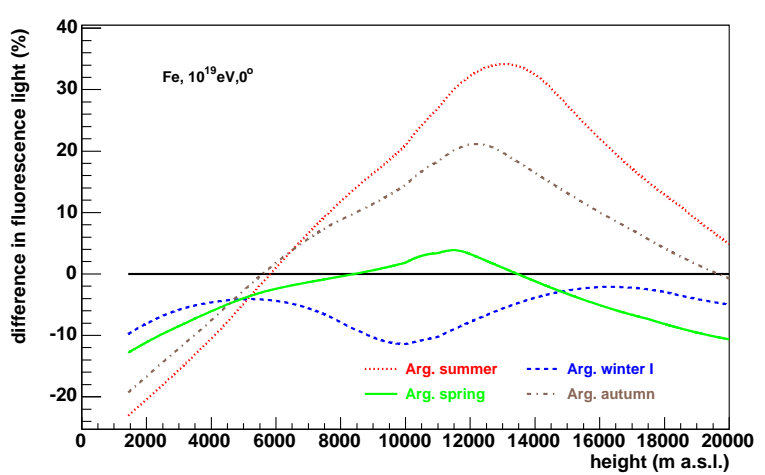

Fig. 18. Difference of the fluorescence light profiles as shown in Fig. 17

$11.1 \%$ during summer, $8.9 \%$ during autumn, $7.3 \%$ during spring, and $6.8 \%$ during winter.

For the $60^{\circ}$ inclined shower, the additional effect due to vapour quenching is smaller and ranges between $1.2 \%$ in summer and $0.2 \%$ in winter. Combining the two effects, the expected light is reduced by $8.4 \%$ during winter, $8.1 \%$ during spring and autumn, and $8.0 \%$ during summer.

\section{Conclusion}

The effects of temperature-dependent collisional quenching cross-sections and of quenching due to water vapour have been studied. Both effects lead to a significant reduction of the fluorescence yield in the lower part of the atmosphere. Applying these calculations to simulated EAS, a distortion of the longitudinal shower development is found. A reduction of the emitted ligth is expected, which varies from about $7 \%$ to $11 \%$ depending on seasonal atmospheric model and on zenith angle of the EAS. Hence, accounting for these effects in the reconstruction of the primary energy of EAS, the primary energy will be increased by this amount as compared with the former model calculations. The position of the shower maximum is hardly shifted, in all atmospheric models the shift is less than $50 \mathrm{~m}$.

Acknowledgement On of the authors (BK) is supported by the German Research Foundation (DFG) under contract KE 1151/1-2. 


\section{References}

[1] C.C. Jui et al. (HiRes Collab.), in: Invited Rapporteur and Highlight Papers, Proceedings of the 26th International Cosmic Ray Conference, Salt Lake City, USA, (2000), 370

[2] J. Abraham et al. (Pierre Auger Collab.), Nucl. Instr. Methods Phys. Res. A523, (2004), 50

[3] M. Fukushima, Prog. Theor. Phys. Suppl. 151, (2003), 206

[4] B. Keilhauer, J. Blümer, R. Engel, H.O. Klages, Astropart. Phys. 25, (2006), 259; astro-ph/0511153 (2005)

[5] A.E. Grün, E. Schopper, Z. Naturforschg. 9a, (1954), 134

[6] P. Privitera for the AirFly Collaboration, in: Proceedings of the 30th International Cosmic Ray Conference, Merida, Mexico, (2007)

[7] A.N. Bunner, Cosmic Ray Detection by Atmospheric Fluorescence, Ph.D. Thesis, Cornell University, Ithaca, NY, USA, (1967)

[8] M. Ave et al. (AirFly Collab.), Astropart. Phys. 28, (2007), 41; astro-ph/0703132 (2007)

[9] A. Morozov, R. Krücken, J. Wieser, A. Ulrich, Eur. Phys. J. D33, (2005), 207

[10] F. R. Gilmore, R. R. Laher, P. J. Espy, J. Phys. Chem. Ref. Data 21, (1992), 1005

[11] J. T. Fons, R. S. Schappe, C. C. Lin, Phys. Rev. A53, (1996), 2239

[12] B. Keilhauer, J. Blümer, R. Engel, H.O. Klages, M. Risse, Astropart. Phys. 22, (2004), 249

[13] J. Knapp, D. Heck, Extensive Air Shower Simulation with CORSIKA: A User's Guide, Report KfK 5196B, Kernforschungszentrum Karlsruhe, (1993); for an up-todate version see http://www-ik.fzk.de/corsika

[14] J. Blümer et al. for the Pierre Auger Collaboration, Proc. 29th Int. Cos. Ray Conf., Pune, India, Vol. 7, p. 123, (2005); astro-ph/0507275. (2005)

[15] T. Waldenmaier, Spectral resolved measurement of the nitrogen fluorescence yield in air induced by electrons, Ph.D. Thesis, University of Karlsruhe, Germany, (2006) 